\title{
A Novel Optical Design Enabling Lightweight and Large Field-Of-View Head-Mounted Microscopes
}

\author{
J. R. Scherrer*1, Galen F. Lynch¹, Jie J. Zhang ${ }^{1}$, Michale S. \\ $\mathrm{Fee}^{\dagger 1}$
}

*equal contribution

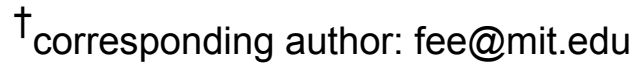

${ }^{1}$ Department of Brain and Cognitive Sciences, McGovern Institute for Brain Research, Massachusetts Institute of Technology, Cambridge, MA 02139, USA

\begin{abstract}
We present a novel fluorescence microscope light path that enables imaging of over a thousand neurons in freely-behaving mice and imaging in juvenile songbirds. The light path eliminates traditional illumination optics, allowing for head-mounted microscopes that have both a lower weight and a larger field-of-view (FOV) than previously possible. Using this light path, we designed two microscopes: one optimized for field-of-view ( 4 mm FOV; $1.4 \mathrm{~g}$ ), and the other optimized for weight (1.0 mm FOV; $1.0 \mathrm{~g}$ ).
\end{abstract}

\section{Main Text}

Miniature head-mounted microscopes have expanded the neuroscience toolkit by enabling in vivo optical measurements of neural activity in freely moving animals ${ }^{1-3}$. Such recordings have been used to study the neural basis of behaviors that are difficult or impossible to recapitulate in head-fixed preparations ${ }^{4}$ like social interaction ${ }^{5}$, vocalization ${ }^{6}$, sleep $^{7}$, and navigation ${ }^{8}$. Despite the impact that head-mounted fluorescent microscopes have had in neuroscience, challenges remain in extending this approach to large populations of neurons and to small model organisms. Understanding how behavior is controlled by neural populations across brain regions requires simultaneous recording in thousands of neurons, which until now has only been possible in head-fixed preparations in small animals ${ }^{9,10}$. Performing these experiments in freely moving animals requires microscopes with a larger field-of-view (FOV) than existing technologies, which are limited to recording from several hundred individually resolvable neurons due to their limited FOV. At the same time, imaging in small organisms such as juvenile mice and juvenile songbirds requires devices with lower weights than existing microscopes, which are too heavy to be used in these small animals. Radically new approaches such as computationally reconstructed imaging are a promising potential solution to FOV and weight limitations ${ }^{11,12}$, but have yet to be successfully applied to functional brain imaging. More incremental improvements to microscope designs have both decreased device weight and 
increased $\mathrm{FOV}^{13-16}$, but these devices are still limited by design tradeoffs that constrain either weight or optical performance.

To address these challenges and opportunities, we describe a novel optical pathway that allows for much lighter microscopes with larger fields of view than previously possible, reducing the tradeoff between weight and FOV inherent to previous designs. Most epifluorescence microscopes couple excitation light using a dichroic filter placed between the objective lens and tube lens (Fig. 1a). The added path length due to the dichroic not only increases microscope dimensions, but also necessitates a larger tube lens to accommodate off-axis rays. The large tube lens size means that the complex optics required for cellular resolution and large FOV imaging quickly become prohibitively heavy. Alternatively, microscopes can be optimized for low device weight, but they typically have a limited FOV (around $1 \mathrm{~mm}^{2}$ ), lower resolution, or decreased light-gathering ability. In contrast, our new optical path brings the objective and tube lens closer together, allowing the optical assembly to be more compact without sacrificing optical performance. To do so, we eliminate the dichroic and instead introduce excitation light at the back aperture of the objective lens (Fig. 1b) with a low-profile light guide and coupling prism.

We used our new light path to design two head-mounted microscopes that advance the state-of-the-art in device weight and FOV. The first design (Featherscope) is optimized for the lowest possible weight without sacrificing resolution. It weighs $1.0 \mathrm{~g}$ but does not sacrifice cellular resolution across a $1.0 \times 1.0 \mathrm{~mm}$ FOV, enabling neural recordings in animals that are too small for existing head-mounted microscopes. We used this first microscope to report, for the first time, functional calcium imaging in freely behaving juvenile songbirds during song learning. The second design (Kiloscope) is optimized for maximum FOV while still achieving an extremely low weight. It provides cellular resolution imaging across a $4.8 \times 3.6 \mathrm{~mm}$ FOV in a device weighing only $1.4 \mathrm{~g}$, allowing us to simultaneously record from over a thousand neurons in a freely behaving mouse.

The Featherscope provides cellular resolution imaging in such a lightweight and compact package by combining the novel optical path described above with compact gradient index (GRIN) optics for both the objective and tube lenses (Fig. 1c,d), a lightweight focusing mechanism, and a custom high-density image sensor printed circuit board (PCB). GRIN lenses both reduce device weight and outperform single spherical lens elements (Supp. Fig. 1a). Excitation light is separated from emission light using a combination of an interference filter and an angle-independent organic dye Wratten filter. Instead of using bulky focusing mechanisms such as electrowetting lenses or screw-tightened sleeves, we provide focus adjustment by compressing the image sensor PCB against polyurethane foam using a screw bracket, allowing fine adjustment of the focus over a range of approximately $200 \mu \mathrm{m}$.

The Kiloscope achieves a wide field of view by using the same optical pathway in combination with precision molded plastic aspheric lens assemblies developed for smartphone cameras (Fig. $1 e, f)$. These lens assemblies achieve diffraction-limited resolution across several millimeters in a compact package. Using two such assemblies for both the objective and tube lenses in a "double macro" configuration ${ }^{17}$, we achieve high resolution imaging with near-flat field curvature 
over a large FOV (Supp. Fig. 1b). The high chief ray angle of the lenses was compensated for using an image sensor with a shifted microlens array.

Illumination for both microscope designs is provided by a fiber-coupled $473 \mathrm{~nm}$ laser, which can provide higher power density than an onboard LED. To reduce imaging artifacts caused by laser speckle, we modulate the laser output using an oscillating ground glass diffuser (Supp. Fig. 2). The despeckled laser light is then coupled into a flexible light guide that terminates at a $300 \mu \mathrm{m}$ $90^{\circ}$ prism mounted to the back aperture of the objective lens. The prism-fiber assembly partially occludes the optical path, reducing the overall light collection efficiency by approximately $20 \%$. Such back-aperture occlusion has a minor effect on diffraction limited spot size, and produces a slight increase in vignetting, but preserves the fine detail necessary for neural imaging. We compensate for optical vignetting (Supp. Fig. 3) by increasing the laser illumination intensity at the edges of the FOV relative to the center using an axicon pair placed before the fiber input coupling optics (Supp. Fig. 2).

Both microscope designs use a custom printed circuit board that supports a CMOS image sensor, serializer, and microcontroller. The microcontroller sequences the start-up of the image sensor, acts as a communications bridge between the image sensor and serializer, and digitizes up to two analog inputs (such as electrophysiological recordings) at 10 bits of resolution with a combined sampling rate of $51 \mathrm{kS} / \mathrm{s}$ which is transmitted in parallel with video data (Supp. Fig. 6). Video data is transmitted to a computer using an interface board based on the UCLA miniscope design $^{3}$. For long-term recordings in behaving animals, a custom commutator transmits laser illumination light along with video data, electrophysiology data, and power.

We quantified imaging performance using standard resolution test targets and measurements of the point spread function. The Featherscope has a resolution of approximately $4 \mu \mathrm{m}$ at the center of its field of view, which decreases to $7 \mu \mathrm{m}$ at the edges (Fig. 2a). The resolution is primarily limited by the astigmatism of the GRIN lenses (Supp. Fig. 1a). The Kiloscope has a resolution of approximately $5 \mu \mathrm{m}$ at the center of its field of view, which decreases to about $6 \mu \mathrm{m}$ at the edges (Fig. 2d), and is primarily limited by the pixel size of the image sensor we use. We then used a higher resolution image sensor to test the Kiloscope optics alone and achieved 2 $\mu \mathrm{m}$ resolution (Supp. Fig. 5). Both microscope designs had cellular resolution in neural tissue across the entire FOV (Fig. 2) and sufficiently small field curvature for typical imaging depths (Supp. Fig. 4).

To demonstrate the utility of the Featherscope in small animals, we recorded calcium activity in a freely behaving juvenile zebra finch during the early stages of song production - previously impossible due to the small size of these juvenile birds. We targeted our recordings to the lateral magnocellular nucleus of the anterior nidopallium (LMAN), a brain region necessary for the earliest stages of song production ${ }^{18}$. After virally expressing GCaMP6f in LMAN, we implanted a GRIN relay lens-prism assembly at the anterior edge of LMAN and let the bird recover. We then installed the Featherscope on the bird's head and allowed the bird to freely behave for several days while recording from LMAN triggered on song (Supp. Video 1). We analyzed the data using EXTRACT, a robust estimation algorithm for extracting microendoscopic calcium activity ${ }^{19}$, 
and extracted 46 neurons (Fig. 3e,f). The activity patterns of almost all neurons were consistent with previous observations of LMAN activity during song (Fig. 3g,h) ${ }^{20}$.

To demonstrate the performance of the Kiloscope, we used the microscope to simultaneously record more than a thousand neurons in the cortex of a freely behaving mouse (Fig. 3a-d). We stereotactically located a region of the mouse brain at coordinates associated with primary visual and somatosensory regions, performed approximately twenty viral injections of GCaMP7f across a $4 \mathrm{~mm}$ diameter region of cortex, and then implanted a $4 \mathrm{~mm}$ diameter glass window in the skull. After letting the mouse recover, we installed the Kiloscope on the mouse's head and allowed it to freely explore a circular maze while recording calcium activity for 12 minutes (Supp. Video 2). We then analyzed the data using EXTRACT and extracted 1526 footprints. Manual curation yielded 1161 footprints that exhibited the spatial profile and temporal fluctuations characteristic of neuronal calcium signals in cortex ${ }^{21}$.

Overall, these microscopes afford the opportunity to record populations of neurons in animals previously inaccessible to functional imaging, and to acquire data in larger populations of neurons than previously possible with head-mounted microscopes. In recent years, comparative neuroscience in small model organisms such as food-caching birds, tree shrews, singing mice, and bats has yielded many discoveries ${ }^{22-25}$. We anticipate that more sophisticated miniaturized open-source tools will allow for further progress using these animals. Simultaneous recording in thousands of neurons has also allowed researchers to understand how activity within and across populations of neurons contributes to behavior ${ }^{9,10}$. Recording similarly large populations of neurons in freely moving animals will advance our understanding of how populations of neurons underlie natural behaviors, such as navigation and social interaction, that are difficult to study in head-fixed preparations.

\section{References}

1. Ghosh, K. K. et al. Miniaturized integration of a fluorescence microscope. Nat. Methods 8, 871-878 (2011).

2. Aharoni, D. \& Hoogland, T. M. Circuit Investigations With Open-Source Miniaturized Microscopes: Past, Present and Future. Front. Cell. Neurosci. 13, 141 (2019).

3. Cai, D. J. et al. A shared neural ensemble links distinct contextual memories encoded close in time. Nature 534, 115-118 (2016).

4. Aghajan, Z. M. et al. Impaired spatial selectivity and intact phase precession in two-dimensional virtual reality. Nat. Neurosci. 18, 121-128 (2015).

5. Li, Y. et al. Neuronal Representation of Social Information in the Medial Amygdala of Awake Behaving Mice. Cell 171, 1176-1190.e17 (2017).

6. Roberts, T. F. et al. Identification of a motor-to-auditory pathway important for vocal learning. Nat. Neurosci. 20, 978-986 (2017).

7. Chen, K.-S. et al. A Hypothalamic Switch for REM and Non-REM Sleep. Neuron 97 , 1168-1176.e4 (2018).

8. Ziv, Y. et al. Long-term dynamics of CA1 hippocampal place codes. Nat. Neurosci. 16, 264-266 (2013). 
9. Steinmetz, N. A., Zatka-Haas, P., Carandini, M. \& Harris, K. D. Distributed coding of choice, action and engagement across the mouse brain. Nature 576, 266-273 (2019).

10. Chen, J. L., Voigt, F. F., Javadzadeh, M., Krueppel, R. \& Helmchen, F. Long-range population dynamics of anatomically defined neocortical networks. Elife 5, e14679 (2016).

11. Adams, J. K. et al. Single-frame 3D fluorescence microscopy with ultraminiature lensless FlatScope. Sci. Adv. 3, e1701548 (2017).

12. Xue, Y., Davison, I. G., Boas, D. A. \& Tian, L. Single-shot 3D wide-field fluorescence imaging with a Computational Miniature Mesoscope. Sci. Adv. 6, eabb7508 (2020).

13. de Groot, A. et al. NINscope, a versatile miniscope for multi-region circuit investigations. Elife 9, e49987 (2020).

14. Scott, B. B. et al. Imaging Cortical Dynamics in GCaMP Transgenic Rats with a Head-Mounted Widefield Macroscope. Neuron 100, 1045-1058.e5 (2018).

15. Rynes, M. L. et al. Miniaturized head-mounted microscope for whole-cortex mesoscale imaging in freely behaving mice. Nat. Methods 18, 417-425 (2021).

16. Leman, D. P. et al. Large-scale cellular-resolution imaging of neural activity in freely behaving mice. bioRxiv (2021) doi:10.1101/2021.01.15.426462.

17. Switz, N. A., D’Ambrosio, M. V \& Fletcher, D. A. Low-Cost Mobile Phone Microscopy with a Reversed Mobile Phone Camera Lens. PLoS One 9, 1-7 (2014).

18. Aronov, D., Andalman, A. S. \& Fee, M. S. A Specialized Forebrain Circuit for Vocal Babbling in the Juvenile Songbird. Science 320, 630-634 (2008).

19. Inan, H. et al. Fast and statistically robust cell extraction from large-scale neural calcium imaging datasets. bioRxiv (2021) doi:10.1101/2021.03.24.436279.

20. Ölveczky, B. P., Andalman, A. S. \& Fee, M. S. Vocal Experimentation in the Juvenile Songbird Requires a Basal Ganglia Circuit. PLOS Biol. 3, e153 (2005).

21. Glas, A., Hübener, M., Bonhoeffer, T. \& Goltstein, P. M. Benchmarking miniaturized microscopy against two-photon calcium imaging using single-cell orientation tuning in mouse visual cortex. PLoS One 14, 1-18 (2019).

22. Payne, H. L., Lynch, G. F. \& Aronov, D. Neural representations of space in the hippocampus of a food-caching bird. Science 373, 343-348 (2021).

23. Dimanico, M. M. et al. Aspects of tree shrew consolidated sleep structure resemble human sleep. Commun. Biol. 4, 722 (2021).

24. Okobi, D. E., Jr., Banerjee, A., Matheson, A. M. M., Phelps, S. M. \& Long, M. A. Motor cortical control of vocal interaction in neotropical singing mice. Science 363, 983-988 (2019).

25. Finkelstein, A. et al. Three-dimensional head-direction coding in the bat brain. Nature 517, 159-164 (2015).

\section{Acknowledgements}

We thank Daniel Aharoni and Jonathan Newman for many helpful discussions and for their technical expertise. In addition we thank Matthew Wilson, Jonathan Newman, Jakob Voigts, Nader Nikbakht, Andrew Bahle, Michael Happ, Alyx Tanner, and Joergen Kornfeld for comments on the manuscript. We thank Wei Guo for providing transgenic mice. M.S.F. acknowledges funding through the McKnight Foundation. J.R.S. acknowledges funding through the Harold and 
Ruth Newman Family Hertz Graduate Fellowship. G.F.L. acknowledges funding through the Simons Foundation (grant 542977ASPI). J.J.Z. acknowledges funding through the NIH (R21 EY028381-01).

\section{Competing Interests}

J.R.S., G.F.L., and M.S.F. are listed as inventors on a patent application for this technology.

\section{Data Availability}

The imaging data presented in this paper is available from the corresponding author upon reasonable request. 

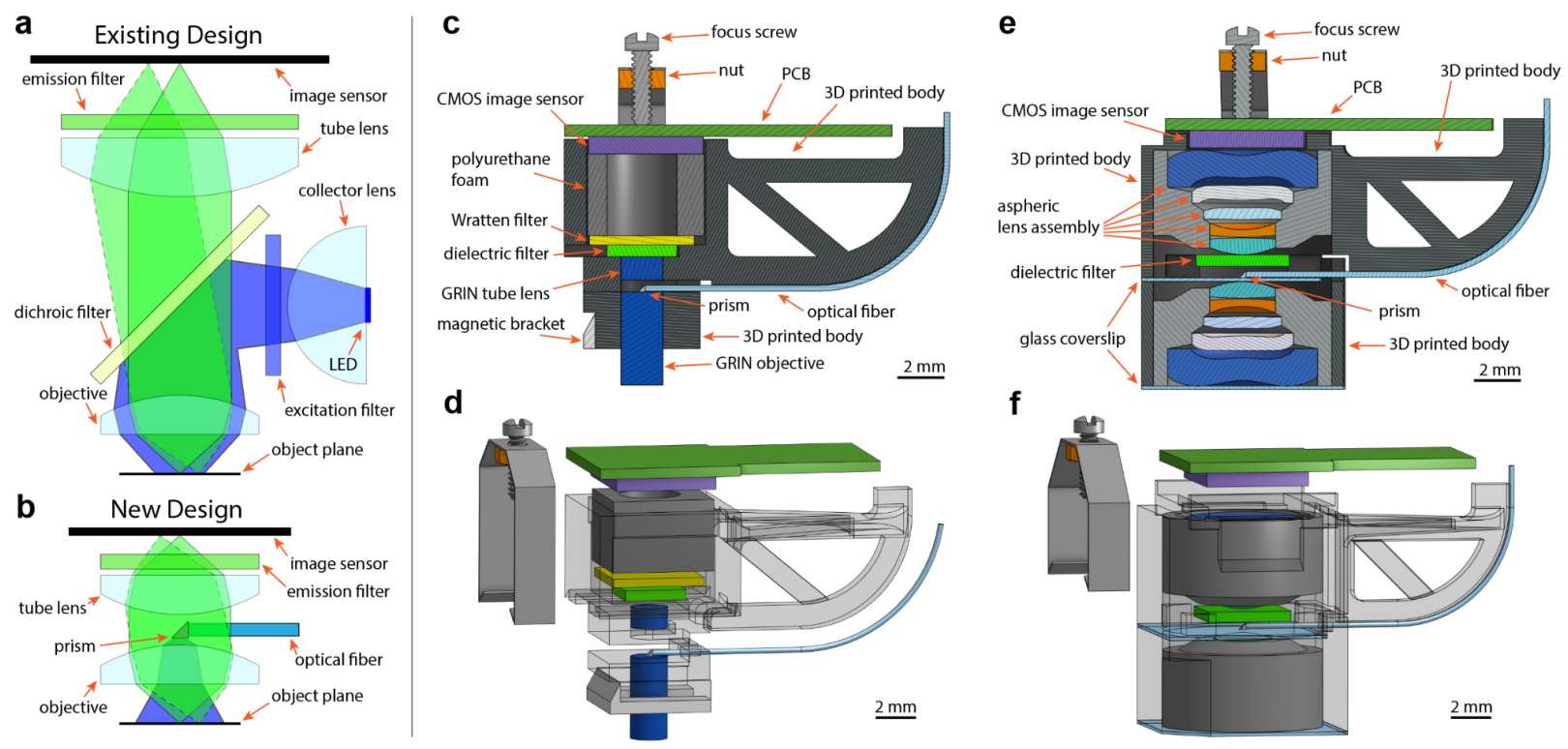

Figure 1 | Design of head-mounted miniature microscopes. a, Schematic representation of optical path for standard head-mounted microscopes and $\mathbf{b}$, for the microscopes in this paper. Ray traces are the excitation light path (blue) and emission light paths for points on-axis (solid green) and off-axis (dashed green). c, cross-section and d, explosion diagrams for the Featherscope. e, cross-section and f, explosion diagrams for the Kiloscope. 


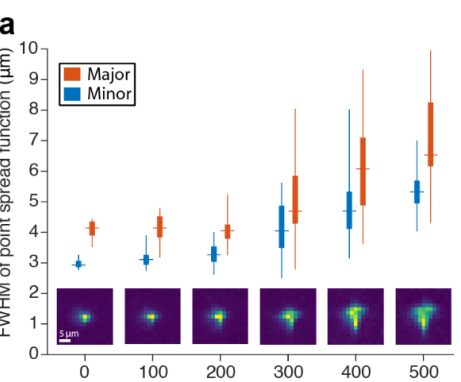

b
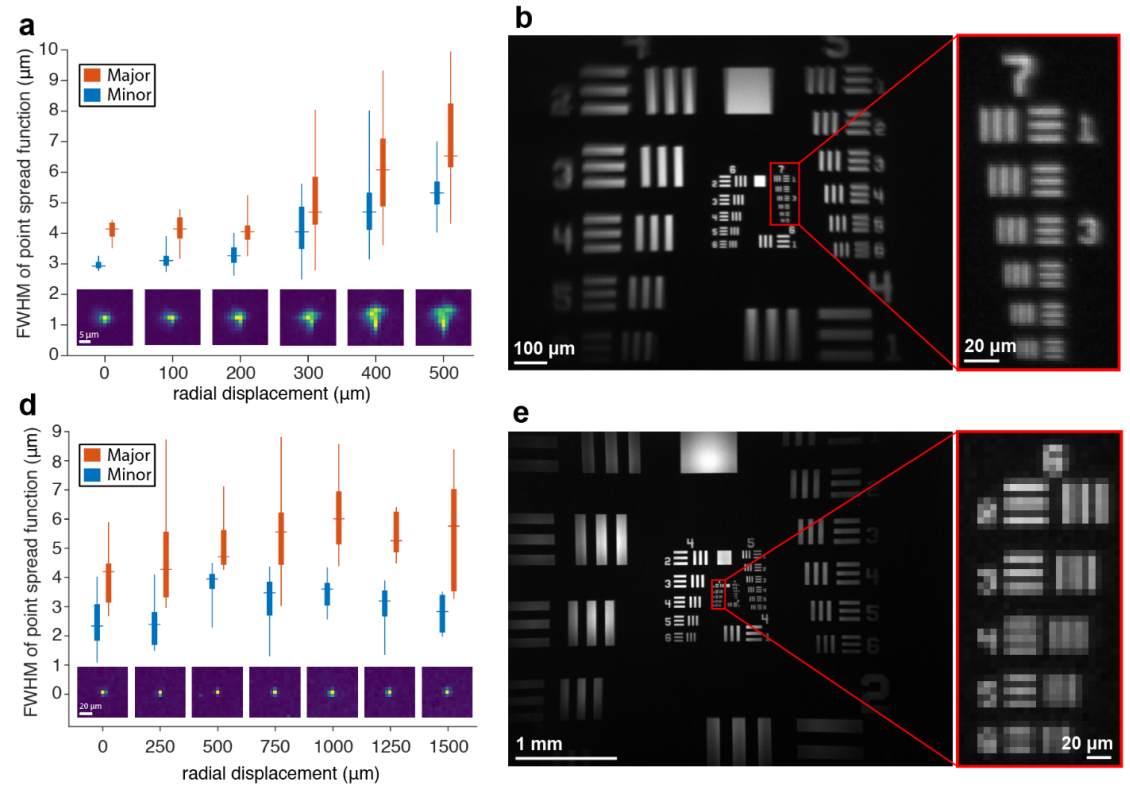

e

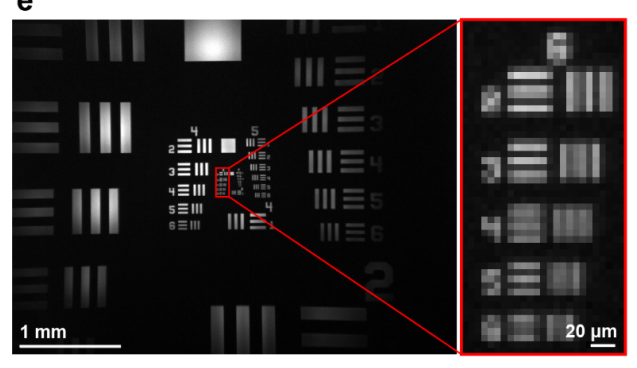

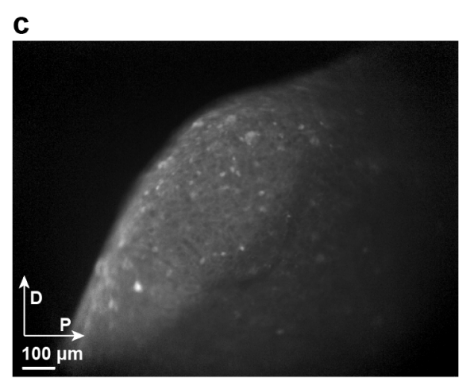

$\mathbf{f}$

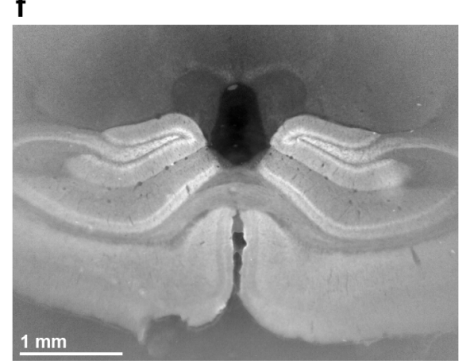

Figure 2 | Optical performance measurements. a, Measurements of the Featherscope's point spread function using $1 \mu \mathrm{m}$ fluorescent beads. Orange and blue data points are measured along the major and minor axes, respectively. The horizontal line represents the median, box edges are the 25th and 75th percentile, and the vertical line extends to the minimum and maximum data points. Inset images are of a representative bead at each position in the object plane. b, Image taken with the Featherscope of a USAF 1951 resolution target. The line widths of the largest and smallest elements visible in the inset are $3.91 \mu \mathrm{m}$ and $2.19 \mu \mathrm{m}$, respectively. c, Image taken with the Featherscope of zebra finch brain slice, fixed and stained with anti-GFP antibody. The image is corrected for vignetting. d, Same as in (a) but with the Kiloscope. e, Same as in (b) but with the Kiloscope. The line widths of the largest and smallest elements visible in the inset are $6.96 \mu \mathrm{m}$ and $4.38 \mu \mathrm{m}$, respectively. f, Image taken with the Kiloscope of a fixed coronal brain slice from a Tg(Camk2a-cre)T29-1Stl x Ai95(RCL-GCaMP6f)-D mouse. The image is corrected for vignetting. 

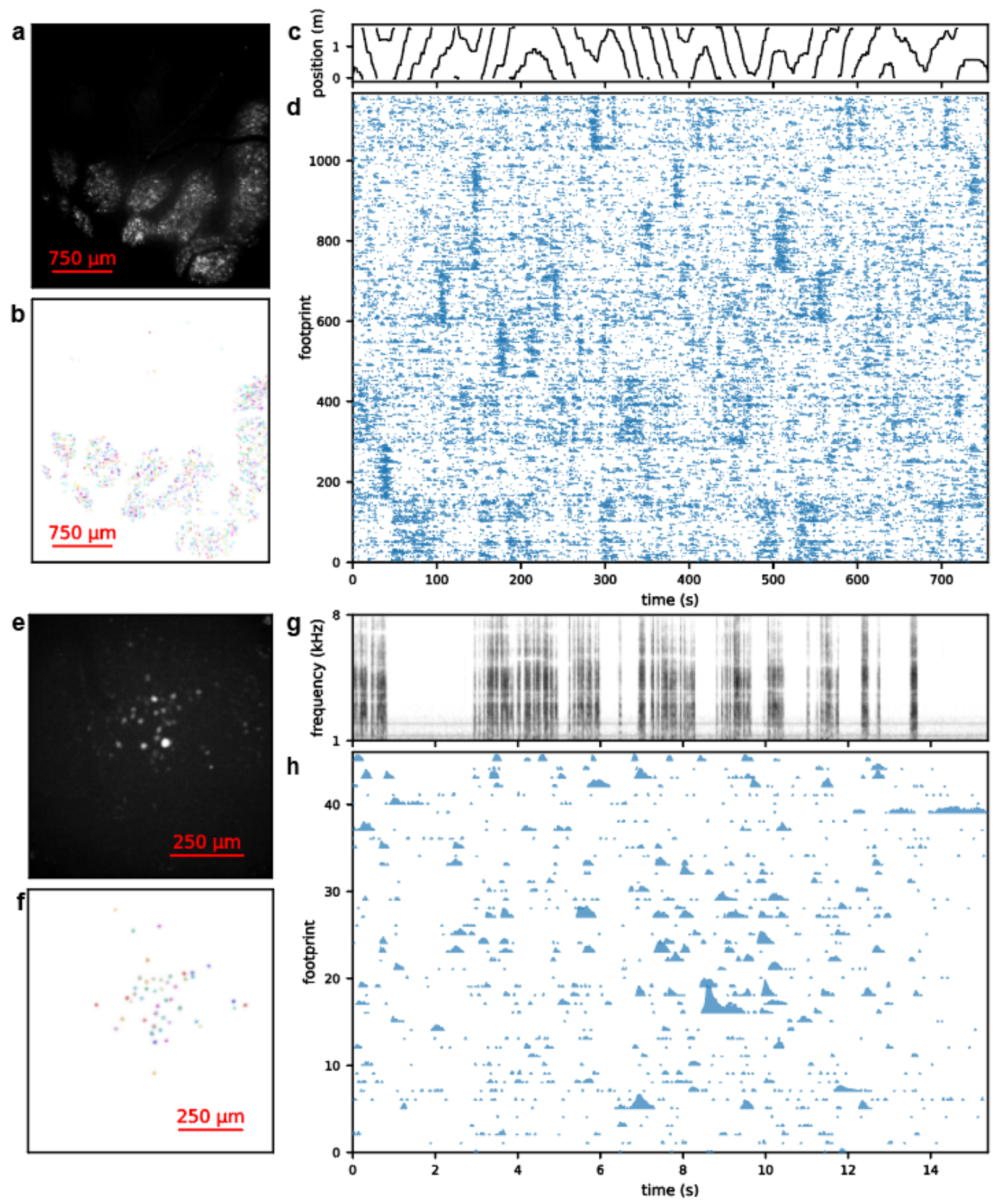

Figure 3 | Demonstration of microscopes in awake freely-behaving animals. a, Maximum intensity projection of fluorescence signal from AAV1-syn-jGCaMP7f expression in mouse cortex imaged during exploration of a circular maze. The anterior portion of the cranial window is at the top, and the medial portion is to the left. $\mathbf{b}$, Spatial footprints of 1161 extracted footprints that were identified as putative neurons in the raw video. $\mathbf{c}$, Linear position along track and $\mathbf{d}$ 
corresponding calcium activity for each of the footprints in (b), sorted by NMF (non-negative matrix factorization) component with activations close to zero not shown. e, Maximum intensity projection of fluorescence signal from AAV9-CAG-GCaMP6f expression in the LMAN of a 57-day old zebra finch imaged during song production. The dorsal portion of the FOV is at the top, and the medial portion is to the left. $\mathbf{f}$, Spatial footprints of 46 neurons extracted using EXTRACT. $\mathbf{g}$, Spectrogram of a single bout of juvenile song and $\mathbf{h}$, corresponding calcium activity for each of the footprints in (f) with activations close to zero not shown. 
a
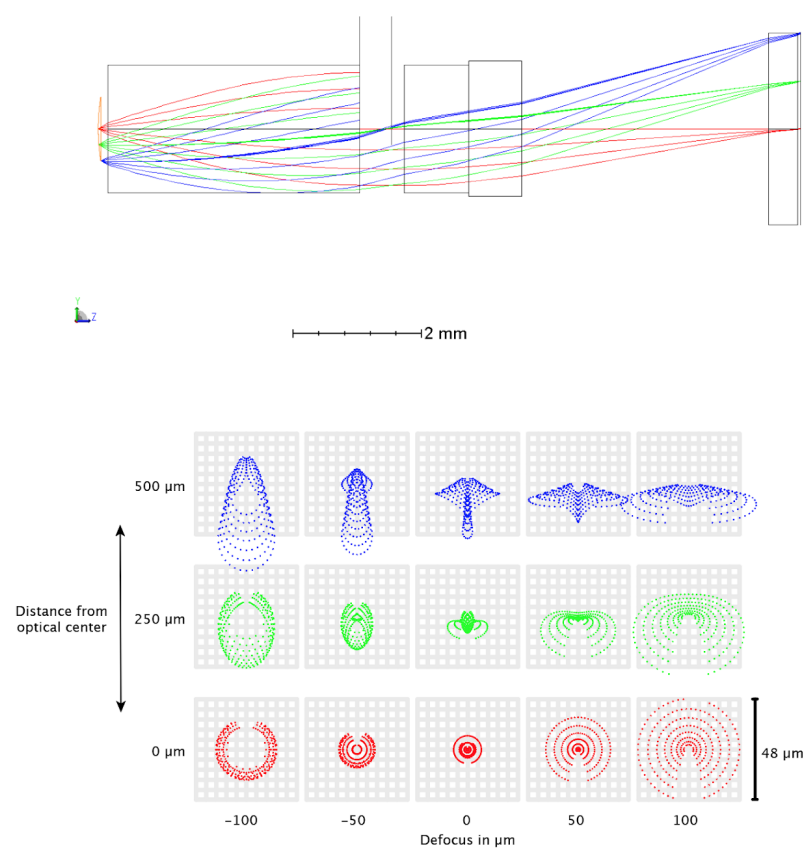

b
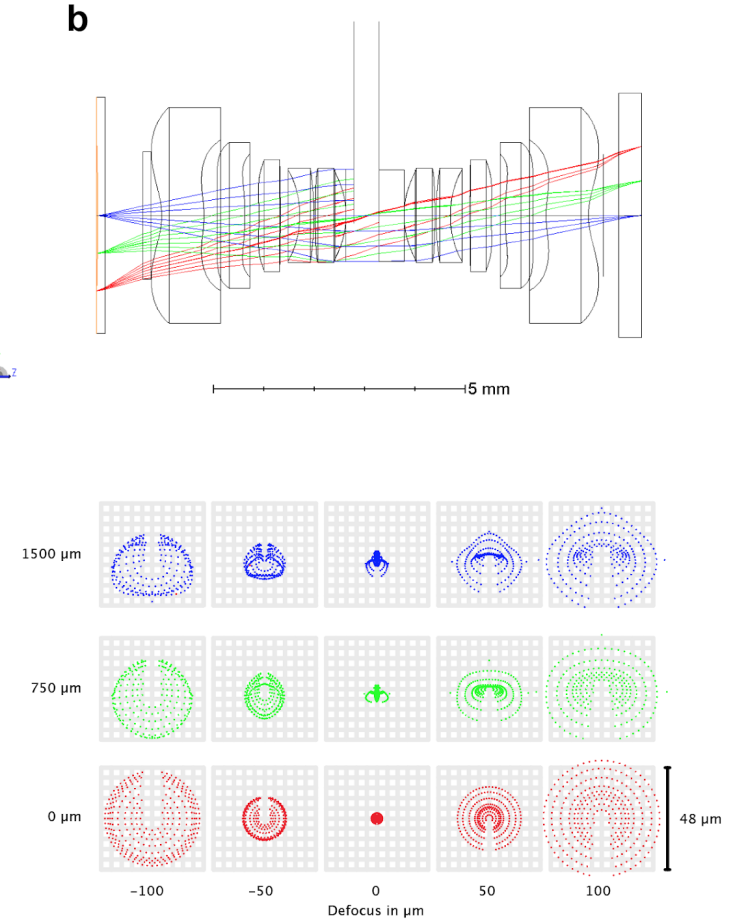

Supplemental Figure 1 | Ray trace analysis of optical assemblies. a, Ray trace simulation of Featherscope lens assembly (above) and point spread function calculated at the image plane (below). The grid spacing is equal to the pixel width. The cross-shaped behavior of the PSF at the edges of the field of view is characteristic of astigmatic aberrations. $\mathbf{b}$, Same ray trace simulation for the Kiloscope lens assembly. 


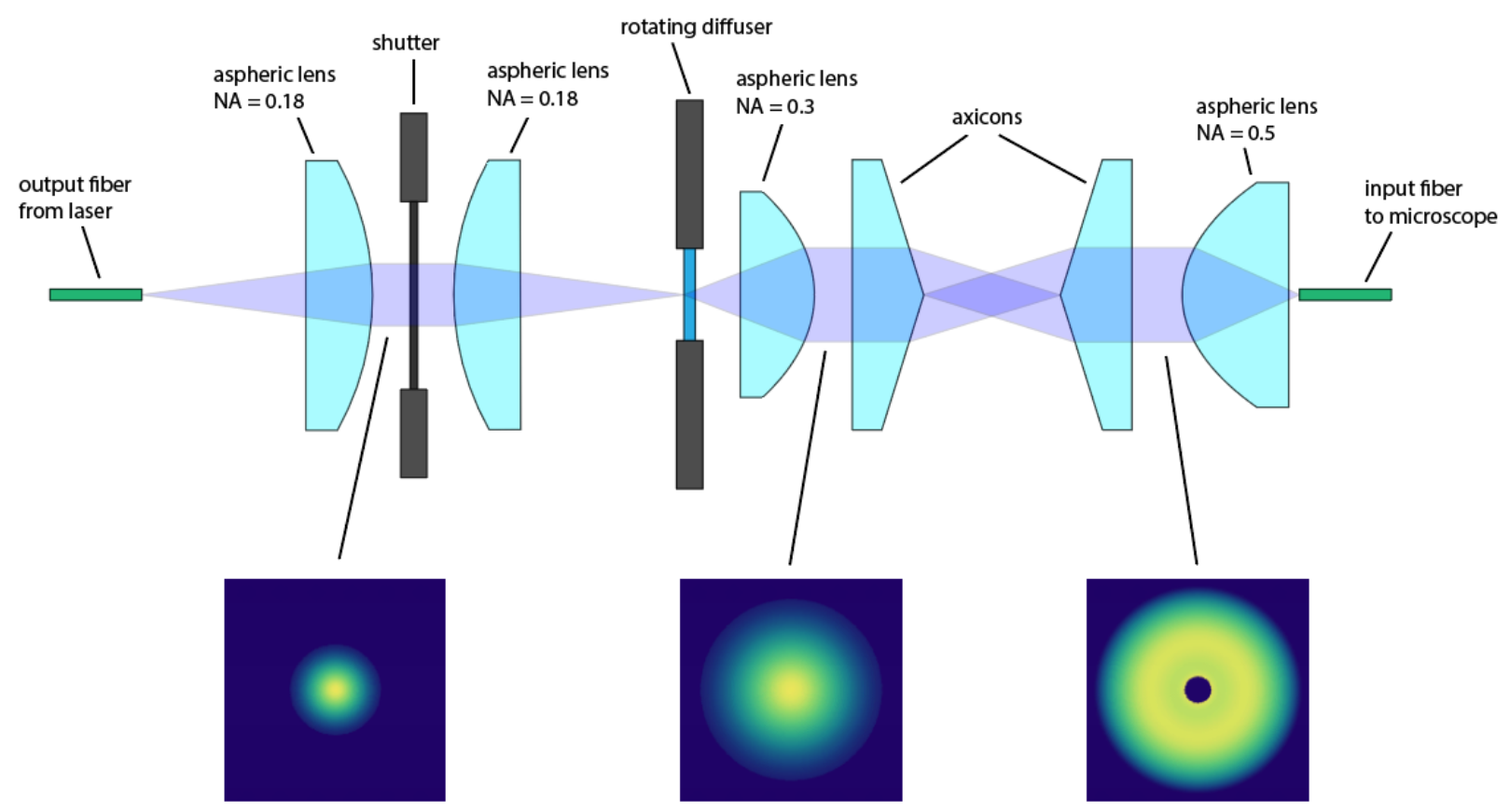

Supplemental Figure 2 | Illumination coupling optics. This optical assembly adapts the output NA of the laser to the NA of the microscope objective, scrambles the modes to prevent illumination speckle, flattens the illumination profile, and provides computer control over illumination to prevent photobleaching. First, a pair of aspheric lenses relays laser light through a computer-controlled shutter onto a rotating diffuser that adds a random phase modulation to the beam. Then, another aspheric lens collimates the output of the diffuser through a pair of axicons that radially invert the beam intensity profile, thereby flattening it. Finally, a high NA aspheric lens couples the beam into the input fiber of the microscope. The beam profile is illustrated below at various points in the assembly. Spacing between the axicons was adjusted for optimal microscope performance, and it was noted that the optimal configuration resulted in a central dark spot in the output beam. This spot is not present at the object plane due to imperfections in the fiber. 

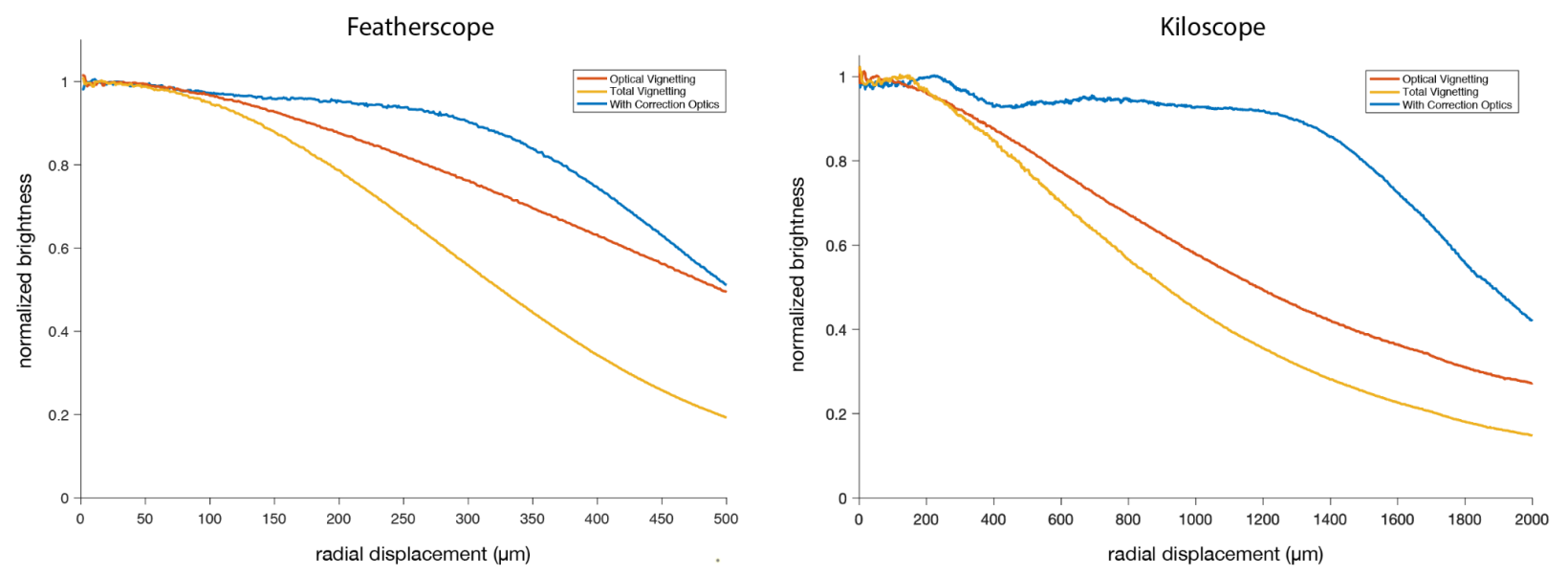

Supplemental Figure 3 | Vignetting correction. Radial profile of the signal collected while imaging a thin layer of fluorescent dye using different optical configurations. Optical vignetting (orange) illustrates the effects of light losses within the microscope optics and was measured by illuminating the dye with a diffuse light source opposite the objective. Total vignetting (yellow) illustrates the decrease in fluorescent signal caused by the combination of optical vignetting and a Gaussian-shaped excitation light profile. The curve with correction optics (blue) illustrates the flat signal intensity profile that can be achieved with an axicon pair that increases the excitation intensity at the edges of the field of view. 


\section{Field Curvature}

Featherscope

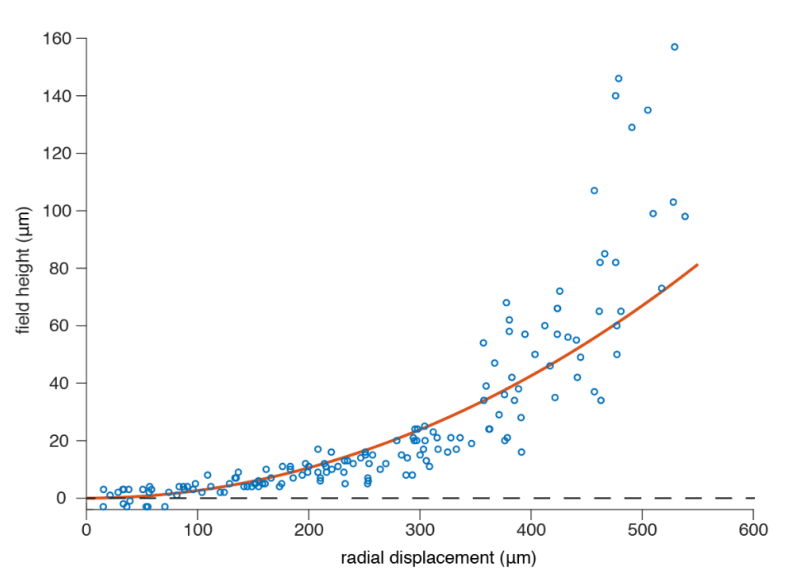

Kiloscope

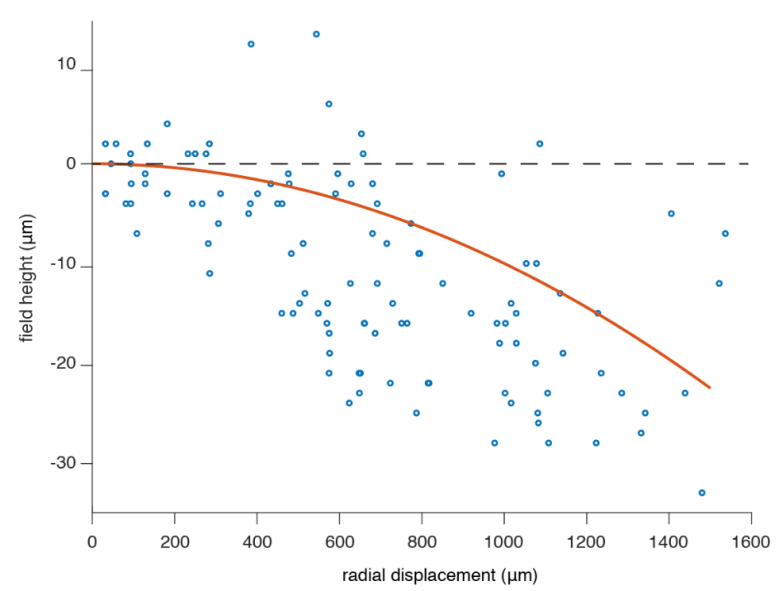

\section{Supplemental Figure 4 | Field Curvature Measurements. Results of bead PSF} measurements compared to simulations. Bead height at best focus is plotted in blue for the set of beads used in the PSF measurement (Fig 2a,d). The observed field curvature is compared to simulation results for a spherical surface of best focus, plotted in orange. 


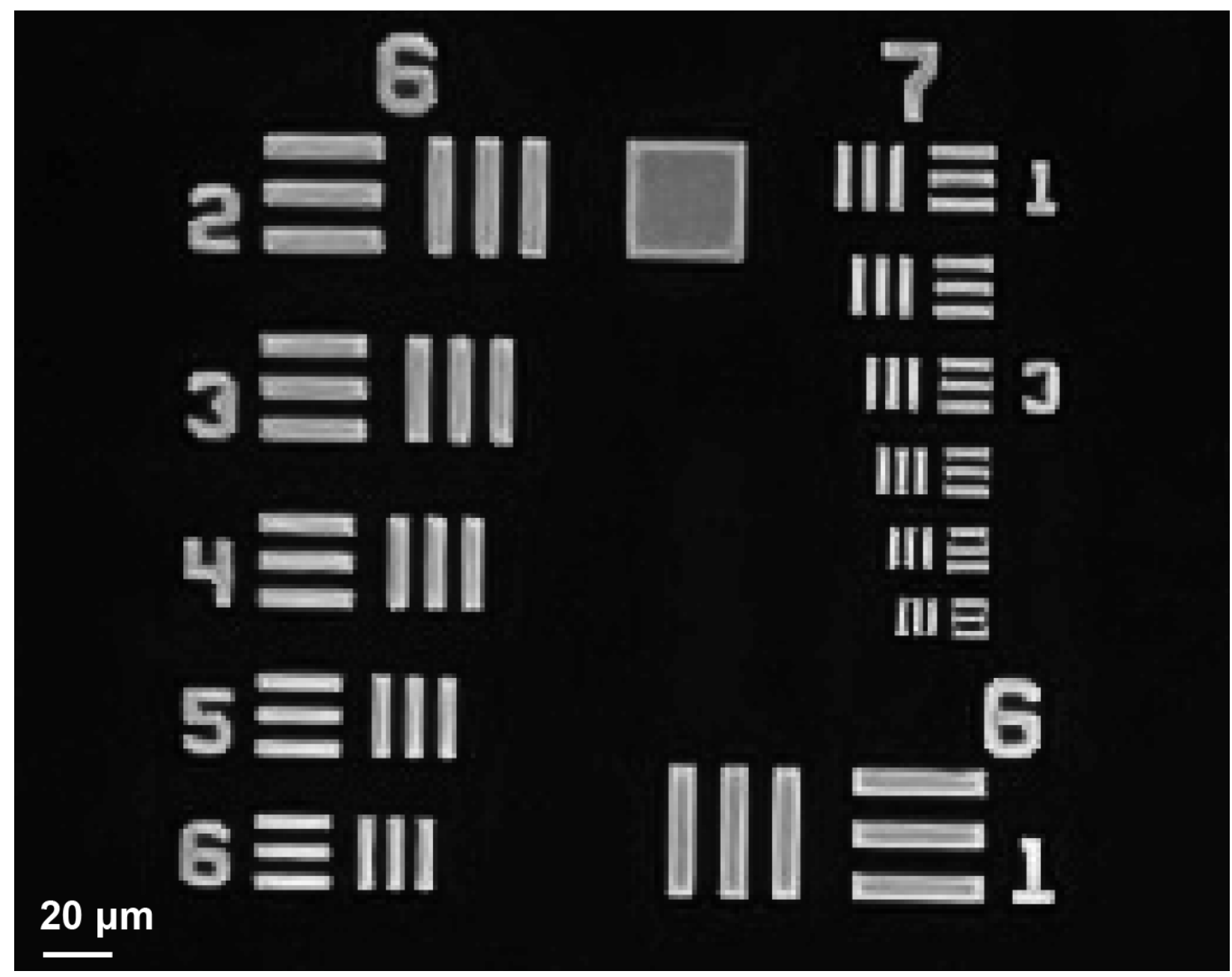

Supplemental Figure 5 | Ultimate attainable resolution of Kiloscope optics. Image of USAF resolution target using a Sony IMX260 image sensor with 1.4 micron pixel width. The smallest element in group 7 is resolvable, indicating a resolution of at least 2.2 microns. 

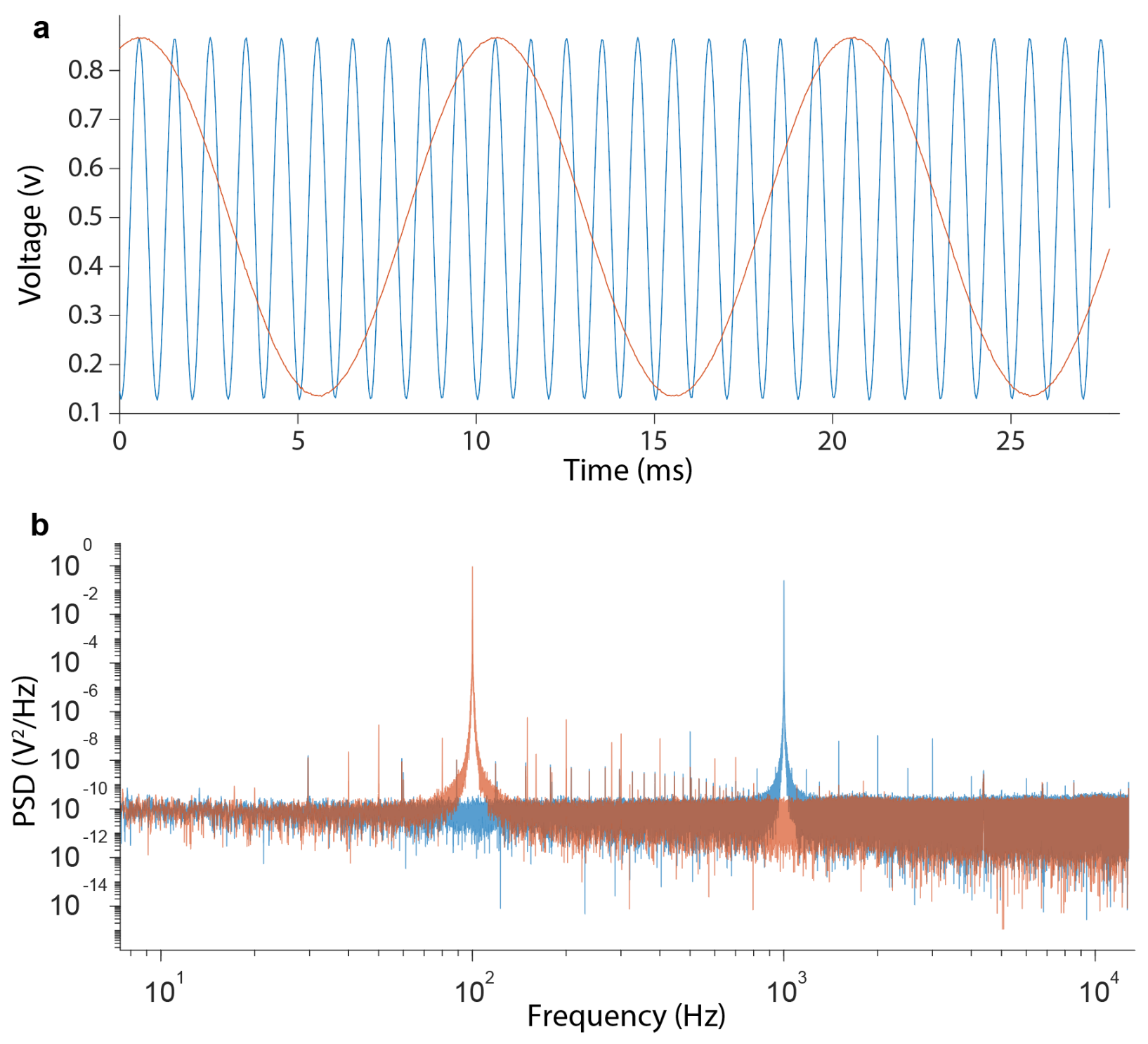

Supplemental Figure 6 | Analog-to-digital converter functionality. a, Time series recording of two test signals at $100 \mathrm{~Hz}$ (orange) and $1000 \mathrm{~Hz}$ (blue) using the onboard analog-to-digital converter, sampling at $25.5 \mathrm{kS} / \mathrm{s}$ for each signal. b. Power spectral density of the test signals. The spectrum was calculated using Thomson's multitaper estimate with Slepian tapers. The time series deviates from a pure tone with a root-mean-square residual of $0.2 \%$ of the full scale, calculated using the full bandwidth of the recorded signal. 


\section{Methods}

\section{Electronics}

The imaging electronics are designed around the Python 480 image sensor (ON Semiconductor NOIP1SN0480A for Featherscope and NOIP1SP0480A for Kiloscope). Our basic data acquisition architecture is modeled after the UCLA Miniscope system ${ }^{1}$. The image sensor outputs data on a 10 bit parallel bus that is sent over coax to a data acquisition (DAQ) board using a serializer and deserializer pair (SERDES; Texas Instruments DS90UB913A-Q1 and DS90UB913A-Q1). The DAQ board uses a Cypress CYUSB3015 controller to send the image data over a USB 3.0 bus to an acquisition computer. The DAQ board is programmed to present itself as a USB video class device (UVC) with uncompressed RGB565 video frames, as 10 bit monochrome frame formats are not supported by UVC. The 10 bit monochrome pixel data occupies the lower 10 bits of the RGB565 pixels (blue and red bits), and conversion from RGB565 to 10 bit monochrome pixel formats is done offline using custom software.

Our image sensor PCB also includes a microcontroller (Atmel ATtiny816) that is used to configure the Python 480, perform power sequencing, provide two channels of analog to digital conversion (ADC), and act as a bridge between the $\mathrm{I} 2 \mathrm{C}$ bus provided by the serializer and the $\mathrm{SPI}$ bus on the image sensor. We use the microcontroller's onboard ADC to read two analog signals. The digitized reads are transmitted as USART serial data over the two most significant bits of the serializer's 12 bit data bus. On the DAQ side, we split the USART bus from the output of the deserializer, convert it to two low-voltage differential signal (LVDS) pairs, and send them off-board via an HDMI connector. We can then acquire these ADC signals with the acquisition computer using an Arduino "Teensy" microcontroller.

The image sensor PCB was designed to be as compact and lightweight as possible. It is a six-layer PCB weighing approximately 300 milligrams. Because it is so small, heat management has a significant effect on image sensor operating temperature and thermal noise. Following the NINScope system, we have disabled the image sensor PLL to reduce power consumption ${ }^{2}$, and have attempted to maximize PCB thermal conductivity by coupling the image sensor and serializer to the ground plane using dense vias. An optional copper fin heat sink can be soldered to the PCB that weighs 150 milligrams and improves SNR by a factor of approximately three. The Kiloscope data was taken with such a heatsink installed, but the Featherscope data was taken without any heatsink.

The DAQ board is a modified version of the UCLA Miniscope v3 DAQ board that uses all twelve bits of the SERDES parallel data bus, rather than the eight in the original design. Two of the four additional bits are routed to the Cypress and used to acquire the full 10 bit output of the image sensor. The other two bits encode ADC reads and are routed to an LVDS converter. From there, the differential signals are sent to an off-board Arduino that sends them over USB to the acquisition computer. 


\section{Optics}

Featherscope: The Featherscope is built from microoptical components cemented into a 3D printed body (Rosenberg Industries LLC) using UV-cure optical cement (Norland Optical Adhesive 61 and 63). The body consists of two subassemblies that are attached once the optics are installed.

The lower subassembly holds the GRIN objective lens (Edmund Optics \#64-537), coupling prism (Edmund Optics \#66-770), and custom fiber bundle (Acrolite, Inc.). Once the objective lens is installed in the body, the prism and fiber bundle are cemented to the back face and covered in a drop of black nail polish (OPI Black Onyx). The nail polish is allowed to dry, and any excess is then carefully trimmed away with a scalpel to expose as much of the back aperture of the objective as possible while maintaining enough thickness ( 50 microns) to block excitation light leakage. A machined magnetic steel flange is cemented to the bottom face of the body to allow for mating with commercially available magnetic baseplates weighing $230 \mathrm{mg}$ (Inscopix).

The upper subassembly holds the tube lens, optical filters, focus mechanism, and image sensor PCB. The tube lens is a GRIN lens (Edmund Optics \#64-537) ground down to a total length of $1.00 \mathrm{~mm}$ (Pioneer Precision Optics). The tube lens, dielectric filter ( $3 \times 3 \times 0.5 \mathrm{~mm}$, Chroma ET525/50m) and Wratten filter (No. 8, Knight Optical \#494FWP) are attached to the body using optical cement. A foam spring is then inserted. The spring is made from compressible polyurethane foam (McMaster \#86375K121) with a $3.0 \mathrm{~mm}$ hole punched using a biopsy punch. The image sensor board is then installed and held in place with a focus bracket. The bracket is constructed from 0.004 " thick 316 stainless steel with a \#000 nut cemented to the underside using JB-Weld epoxy. A hole is punched above the nut and a \#000-120 screw is threaded into the bracket. During normal operation, the screw pushes against the top of the onboard microcontroller and compresses the foam spring.

Once the two subassemblies are built, they are attached together using optical cement. The fiber bundle is then attached to the relief arm of the upper subassembly body using dental floss and potted using 5 minute epoxy (Loctite 1365868). Thin and flexible lines for power (Cooner Wire \#CZ1174) and coaxial data (Cooner Wire \#CW6563) are braided together with the fiber bundle and secured using dental floss.

Kiloscope: the Kiloscope is constructed in a similar manner to the Featherscope using two subassemblies of optics mounted in 3D printed housings. The lower subassembly holds the objective lens, an imaging lens extracted from the rear camera module of a Samsung Galaxy S9 smartphone. These imaging lenses are carefully removed from rear camera replacement modules available from third-party vendors. The lens is installed in the printed housing and enclosed at the top and bottom by two pieces of protective No. 1 coverglass. The fiber-prism assembly is installed at the top coverglass using the same procedure as the Featherscope. 
The upper subassembly holds the dielectric filter, tube lens, focus mechanism, and imaging $\mathrm{PCB}$. The tube lens is identical to the objective lens and is attached directly to the dielectric filter. Compressible sorbothane rubber strips (5000 Durometer, McMaster \#8824T11) are used as focus springs and are inserted on ledges designed into the housing. The PCB is then installed and held in place with a focus bracket similar to that used with the Featherscope. The fiber is secured to the relief arm of the housing and is braided together with the electrical lines. The microscope slides into a custom 3D printed baseplate weighing $180 \mathrm{mg}$.

The Kiloscope exhibits some degree of autofluorescence caused by the plastic optics in the objective lens assembly. However, this fluorescence bleaches out to a negligible background level after an initial exposure period.

\section{Imaging Performance Characterization}

Imaging performance was measured using two methods. The first was using a standard 1951 USAF resolution test target against a fluorescent background. This method has the benefit of intuitive interpretation and broad recognizability, but it is somewhat subjective and does not include information about the resolution of the system at different points in the FOV. Miniature endoscopes such as ours have significant aberrations that vary across the field of view and tend to be minimized at the center, so measurements using resolution test targets at a single position can overestimate the full-field resolution.

Because of these limitations, we decided to quantify the point spread function of our microscopes using an array of beads imaged at various positions and vertical displacements within the FOV. The PSF contains the same information as a two-dimensional modulation transfer function (MTF) measurement taken at various positions, but has the added benefit of being intuitively interpretable for researchers who are not familiar with optical engineering methods.

For the PSF data in Fig. 2, we imaged a slide of 1 micron diameter fluorescent beads at a series of vertical positions spanning 100 microns of depth. We then found the position corresponding to the most focused image for each bead, and fit a two dimensional Gaussian distribution to that image to find an approximate FWHM. We also measured the field curvature by plotting the vertical position of best focus as a function of radial distance (Supp. Fig. 3). We sampled 154 and 112 beads for the Featherscope and Kiloscope analysis, respectively.

\section{Commutator}

Our commutator simultaneously provides power, RF data, and illumination light to our microscopes. We couple DC power and RF data through an electrical slip ring (Moflon MSP1069) similar to existing open source designs (University of Colorado ONE Core ${ }^{3}$ ). We transmit light through an optical fiber threaded through the bore of the slip ring and attached to an optical rotary joint (Thorlabs RJ1) mounted coaxially with the slip ring. We drive the commutator using a motor controlled by a magnetic feedback signal, similar to existing designs ${ }^{4}$. 
The slip ring presents an impedance mismatch for the $50 \mathrm{Ohm}$ transmission line and on its own causes disruptive signal reflections. However, because the slip ring size is a small fraction of the carrier wavelength, we can partially compensate for the mismatch by adding a small tuning capacitor (2-6 pF) in parallel with the slip ring. Using this capacitor, along with boosting the forward channel low frequency gain of the SERDES, we can transmit data through the slip ring without excessive frame dropping.

\section{Coupling Optics}

The illumination coupling optics are based on a set of four aspheric lenses (Thorlabs C280TMD-A, C230TMD-A, and A397-A) that adapt the laser output NA of 0.1 to the higher microscope fiber NA of 0.5. A shutter placed in between the first two lenses provides fast control over illumination to prevent photobleaching while avoiding power instability caused by modulating the laser current.

We reduce speckle in the laser output using an oscillating diffuser (Optotune LSR-3005-17S-VIS) placed at the imaged laser spot between the second and third lenses. This diffuser adds a random time-varying phase to the beam waist that randomizes the speckle pattern in the object plane. The diffuser oscillates at $300 \mathrm{~Hz}$, so a single frame integrates over many of these random patterns, averaging them together and reducing the overall speckle. We do not observe any residual speckle in our in vivo data after despeckling.

The Gaussian output of the laser results in a Gaussian distribution of illumination in the object plane. This distribution combined with the optical vignetting of the microscope results in dim fluorescence signals and thus low SNR at the edges of the FOV. To compensate, we use a pair of axicons (Thorlabs AX1220) between the third and fourth lenses that transforms the Gaussian input into a relatively flat output. The precise shape of the output distribution can be adjusted by varying the distance between the axicons.

\section{Data Analysis}

In vivo imaging data was analyzed using the NoRMCorre and EXTRACT algorithms ${ }^{5,6}$. For the Kiloscope data, we first ran NoRMCorre to correct for brain motion. We then ran EXTRACT using mostly default parameter values but with an increased value for the minimum cellfinding SNR of 2 . This extraction yielded 1526 neurons, which were then manually curated by viewing the mean-subtracted video in the region of each extracted footprint at times corresponding to the peak extracted activity. We scored each neuron by whether it appeared by eye to be a visually distinct point modulated in time and whether it was spatially separated from blood vessels. This manual curation yielded 1161 candidate neurons and is a lower bound on the actual number of neurons in the dataset. The resulting time series were factored using nonnegative matrix factorization (NMF) into eight component factors, and the data in Figure $3 d$ were sorted according to these factors to highlight potential low dimensionality of the data. 
For the Featherscope data, we extracted neurons using the same pipeline, with the exception that image alignment was performed using custom software. The extracted neurons were manually curated in the same way, yielding 46 candidate neurons used in the analysis.

\section{Video Processing}

Example videos were generated using custom Julia software. The fluorescence signal was median filtered over time. For the mouse data (Supp. Video 2), the signal was temporally downsampled by a factor of four. The image levels of both videos were adjusted for display purposes.

Maximum intensity projections (Fig. 3a, e) were calculated using custom Julia software. The fluorescence signal was first median filtered over time and then spatially bandpass filtered. The image levels of each maximum intensity projection were adjusted for display purposes.

\section{Subjects and Calcium Imaging}

Calcium imaging was performed in juvenile male zebra finches (Taeniopygia guttata) 50-70 days post-hatch (dph) during undirected song, and in adult C57/B6 mice during free exploration of a circular maze. Birds and mice were obtained from the Massachusetts Institute of Technology breeding facility (Cambridge, Massachusetts) and Jackson labs, respectively. Video and behavioral data was collected using custom Bonsai workflows ${ }^{7}$. The care and experimental manipulation of the animals were carried out in accordance with guidelines of the National Institutes of Health and were reviewed and approved by the Massachusetts Institute of Technology Committee on Animal Care.

Prior to surgery, birds were isolated from their tutor at $35 \mathrm{dph}$. Birds were anesthetized with $1-2 \%$ isoflurane in oxygen and placed in a stereotaxic apparatus. LMAN was mapped using antidromic identification from RA as previously described ${ }^{8}$, and RA was injected with cholera toxin subunit B (recombinant) Alexa Fluor 647 conjugate (Invitrogen) to retrogradely label LMAN for histological verification. LMAN was then injected with AAV9.CAG.GCaMP6f.WPRE.SV40 ${ }^{9}$ (University of Pennsylvania Viral Core) using a Nanoject II (Drummond). After a period of time to allow the virus to perfuse the neural tissue, we lowered a GRIN prism relay lens (Inscopix) anterior to LMAN, and attached it to the skull using previously described methods ${ }^{10} .10$ days following the injection procedure, birds were anesthetized and a base plate (Inscopix) was attached to the skull.

Mice were anesthetized with 1-2\% isoflurane in oxygen and placed in a stereotaxic apparatus. V1 was localized using stereotaxic coordinates, and a $4 \mathrm{~mm}$ diameter craniotomy was made to expose $\mathrm{V} 1$ as well as brain regions anterior and lateral to it. We then performed a series of injections of AAV1 pGP-AAV-syn-jGCaMP7f-WPRE ${ }^{11}$ (Addgene) with 500-600 $\mu \mathrm{m}$ spacing across the craniotomy. We injected $200 \mathrm{~nL}$ of the virus per site at a depth of $200 \mu \mathrm{m}$ below the dura. A cranial window composed of one $4 \mathrm{~mm}$ diameter coverslip glued to a $5 \mathrm{~mm}$ diameter cover slip (Carolina) was inserted into the craniotomy and attached to the skull. After about two weeks to allow for expression, a custom base plate was attached to the skull. 


\section{Supplemental References}

1. Cai, D. J. et al. A shared neural ensemble links distinct contextual memories encoded close in time. Nature 534, 115-118 (2016).

2. de Groot, A. et al. NINscope, a versatile miniscope for multi-region circuit investigations. Elife 9, e49987 (2020).

3. Slick Passive Electrical Commutator. Optogenetics and Neural Engineering Core, University of Colorado School of Medicine.

https://optogeneticsandneuralengineeringcore.gitlab.io/ONECoreSite/projects/SlickPassi veElectricalCommutator/.

4. Fee, M. S. \& Leonardo, A. Miniature motorized microdrive and commutator system for chronic neural recording in small animals. J. Neurosci. Methods 112, 83-94 (2001).

5. Pnevmatikakis, E. A. \& Giovannucci, A. NoRMCorre: An online algorithm for piecewise rigid motion correction of calcium imaging data. J. Neurosci. Methods 291, 83-94 (2017).

6. Inan, H. et al. Fast and statistically robust cell extraction from large-scale neural calcium imaging datasets. bioRxiv (2021) doi:10.1101/2021.03.24.436279.

7. Lopes, G. et al. Bonsai: an event-based framework for processing and controlling data streams. Front. Neuroinform. 9, 7 (2015).

8. Aronov, D., Andalman, A. S. \& Fee, M. S. A Specialized Forebrain Circuit for Vocal Babbling in the Juvenile Songbird. Science 320, 630-634 (2008).

9. Chen, T.-W. et al. Ultrasensitive fluorescent proteins for imaging neuronal activity. Nature 499, 295-300 (2013).

10. Okubo, T. S., Mackevicius, E. L. \& Fee, M. S. In Vivo Recording of Single-Unit Activity during Singing in Zebra Finches. Cold Spring Harb. Protoc. 2014, pdb.prot084624 (2014).

11. Dana, H. et al. High-performance calcium sensors for imaging activity in neuronal populations and microcompartments. Nat. Methods 16, 649-657 (2019). 